\title{
A GENERALISATION OF A RECENT CHARACTERISATION OF PLANAR GRAPHS
}

\author{
C.H.C, LITTLE
}

\begin{abstract}
Planar graphs have recently been characterised as those which have no strict elegant odd ring of circuits. Here we generalise that result by showing that its dual yields a theorem that is valid for all graphs.
\end{abstract}

\section{Introduction}

A new characterisation of planar graphs appears in [2]. This characterisation hinges on the idea of a ring of circuits. If $C$ is a collection of circuits of a graph $G$ and the edges of $G$ can be directed so that every circuit of $\mathcal{C}$ is a directed circuit, then we say that $\mathcal{C}$ is consistently orientable. The cyclic sequence $c=\left(c_{0}, c_{1}, \ldots, c_{n-1}\right)$ of circuits, where $n \geq 3$, is a ping of circuits in the graph $G$ if

(i) C is consistently orientable,

(ii) $E C_{i} \cap E C_{j} \neq \varnothing$ if and only if $i=j, i \equiv j+1(\bmod n)$ or $i \equiv j-1(\bmod n)$, and

(iii) no edge of $G$ belongs to more than two circuits of $\mathcal{C}$. The ring $C$ above is odd if $n$ is odd, strict if $\left|V C_{i} \cap V C_{j}\right| \leq 1$ whenever $E C_{i} \cap E C_{j}=\emptyset$, and elegant if for each $i \in\{0,1, \ldots, n-1\}$ there is a path $M_{i}$ satisfying the conditions $E M_{i}=E C_{i+1}-E C_{i}$ and $\left|V M_{i} \cap V C_{i}\right|=2$. (Here, and throughout this paper, subscripts are to be

Received 29 October 1982. 
read modulo $n$.)

It is proved in [2] that a graph is planar if and only if it contains no strict elegant odd ring. The proof in [2] has been greatly simplified by Chen [1] using topological considerations. In this paper, we achieve further simplification by dualising some of Chen's ideas, thereby also generalising the result.

Let $G$ be a graph and $X, Y \subseteq V G$. We denote by $[X, Y]$ the set of all edges joining a vertex of $X$ and a vertex of $Y$. If $Y=V G-X$, then $[X, Y]$ is called an edge cut if $X \neq \emptyset$ and $Y \neq \emptyset$. We abbreviate $[X, Y]$ by $\delta(X)$ or $\delta(Y)$. A minimal non-empty edge cut is a bond. It is well known that if $G$ is connected, then $[X, Y]$ is a bond if and only if $G[X]$ and $G[Y]$ are connected. Moreover, any non-empty edge cut is a union of disjoint bonds. In the case where $G$ is a directed graph and every edge of $\delta(X)$ is directed toward the end in $X$, we say that $\delta(X)$ is a directed edge cut.

If $C$ is a collection of bonds of a graph $G$ and the edges of $G$ can be directed so that every bond of $\mathcal{C}$ is directed, then we say that $C$ is consistently orientable. The cyclic sequence $\mathrm{C}=\left(c_{0}, c_{1}, \ldots, c_{n-1}\right)$ of bonds, where $n \geq 3$, is a ring of bonds in the graph $G$ if

(i) $\mathcal{C}$ is consistently orientable,

(ii) $C_{i} \cap C_{j} \neq \emptyset$ if and only if $i=j, i \equiv j+1(\bmod n)$ or $j \equiv j-1(\bmod n)$, and

(iii) no edge of $G$ belongs to more than two bonds of $\mathcal{C}$.

Let $A$ and $B$ be bonds of $G$, where $A=[X, Y]$. Then a proper subset $P$ of $B$ is a $B$-chord of $A$ if $P$ is a bond of $G[X]$ or $G[Y]$. Note that $P$, being a proper subset of $B$, cannot be a bond of $G$. Hence if $P=[S, T]$ and $P$ is a bond of $G[X]$, then $[Y, S] \neq \emptyset$ and $[Y, T] \neq \emptyset$. Similarly if $P$ is a bond of $G[Y]$, then $[X, S] \neq \emptyset$ and $[X, T] \neq \emptyset$.

The ring $c=\left(c_{0}, c_{1}, \ldots, c_{n-1}\right)$ of bonds is odd if $n$ is odd, even if $n$ is even, strict if there do not exist distinct bonds $A, B, C$ satisfying the conditions $B \in \mathcal{C}, C \in \mathcal{C}, B \cap C=\emptyset, A \subseteq B \cup C$, and elegant if for each $i$ there exists a unique $c_{i+1}$-chord of $c_{i}$. It is 
easily seen that if $G$ is planar the dual of a strict elegant odd ring of circuits is a strict elegant odd ring of bonds. Hence no planar graph can contain a strict elegant odd ring of bonds. It is our purpose here to generalise this result to non-planar graphs by proving the following theorem.

THEOREM. In any graph, every strict elegant ming of bonds is even.

\section{Preliminary lemmas}

Throughout the rest of this paper, we let $C$ be a ring $\left(c_{0}, C_{1}, \ldots, c_{n-1}\right\}$ of bonds in a graph $G$. Furthermore, for each $i$ we will write $C_{i}=\left[A_{i}, B_{i}\right]$.

LEMMA 1. There is a component $D$ of $G$ such that $\bigcup_{i=0}^{n-1} C_{i} \subseteq E D$.

Proof. Let $D$ be a component of $G$ such that $E D \cap C_{0} \neq \emptyset$. Since $C_{0}$ is a bond, we have $C_{0} \subseteq E D$. Proceeding by induction, assume that $k-1$

$\bigcup_{i=0}^{U} C_{i} \subseteq E D$ for some $k>0$. Since $C_{k-1} \cap C_{k} \neq \emptyset$, it follows that $E D \cap C_{k} \neq \emptyset$; hence $C_{k} \subseteq E D$ since $C_{k}$ is a bond. The result follows by induction.

Thus we henceforth assume without loss of generality that $G$ is connected.

LEMMA 2. For any $i$ and any $e \in C_{i+1}-C_{i}$, there exists a $C_{i+1}$ chord of $c_{i}$ which contains $e$.

Proof. Without loss of generality, let $e \in\left[A_{i}, A_{i}\right]$. Then $\left[A_{i} \cap A_{i+1}, A_{i} \cap B_{i+1}\right]$ is a non-empty edge cut of $G\left[A_{i}\right]$ and so a union of bonds of $G\left[A_{i}\right]$. Since $C_{i} \cap C_{i+1} \neq \emptyset$, these bonds are proper subsets of $c_{i+1}$, and hence $c_{i+1}$-chords of $c_{i}$. One of them contains $e$.

LEMMA 3. If $\mathrm{C}$ is elegant, then for each $i$ there is a unique $c_{i}-$ chord of $c_{i+1}$. 
Proof. Let $P=[X, Y]$ be the unique $C_{i+1}$-chord of $C_{i}$, and without loss of generality let $P \subseteq\left[A_{i}, A_{i}\right]$. By Lemma 2 , any $e \in C_{i+1}-C_{i}$ must belong to $P$. Hence $P=C_{i+1}-C_{i}$, and it follows immediately that we may assume $C_{i+1}=P \cup\left[X, B_{i}\right]$ without loss of generality. Since $G\left[B_{i}\right]$ and $G[Y]$ are connected and $\emptyset \subset\left[Y, B_{i}\right] \subset C_{i}$, it follows that $\left[Y, B_{i}\right]$ is a $C_{i}$-chord of $C_{i+1}$, and it is clearly the only such chord.

LEMMA 4. Let $\mathrm{C}$ be strict and elegant. Then for all $i, j, k \in\{0,1, \ldots, n-1\}$, either $\left(C_{j} \cup C_{k}\right)-C_{i} \subseteq\left[A_{i}, A_{i}\right]$ or $\left(C_{j} \cup C_{k}\right)-C_{i} \subseteq\left[B_{i}, B_{i}\right]$.

Proof. Without loss of generality, let $i=0$ and $j \leq k$. We shall show first that either $C_{j}-C_{0} \subseteq\left[A_{0}, A_{0}\right]$ or $C_{j}-C_{0} \subseteq\left[B_{0}, B_{0}\right]$. This statement is trivial if $j=0$; suppose therefore that $j>0$.

Case I. Suppose $1<j<n-1$. Then $C_{0} \cap C_{j}=\emptyset$ by (ii).

Suppose that $C_{j} \cap\left[A_{0}, A_{j}\right] \neq \emptyset$ and $C_{j} \cap\left[B_{0}, B_{0}\right] \neq \emptyset$. Then $A_{0} \cap A_{j}, A_{0} \cap B_{j}, B_{0} \cap A_{j}$ and $B_{0} \cap B_{j}$ are all non-empty. Let $D$ be a component of $G\left[A_{0} \cap A_{j}\right]$. Thus $\delta(V D) \subseteq C_{0} \cup C_{j}$.

We show next that $\delta(V D)$ is a bond. Since $D$ is connected, it suffices to demonstrate that $G-V D$ is connected. As $C_{j}$ is a bond, $G\left[B{ }_{j}\right]$ must be connected. Because $V D \subseteq A_{j}$, there must therefore be a component $X$ of $G-V D$ for which $B_{j} \subseteq V X$. If $D^{\prime}$ is any component of $G\left[A_{0} \cap A_{j}\right]$ other than $D$, then $\left[V D^{\prime}, A_{0} \cap A_{j}\right] \neq \emptyset$ because $G\left[A_{0}\right]$ must be connected; hence $V D^{\prime} \subseteq V X$. Finally, since $B_{0} \cap B_{j} \neq \varnothing$ and $G\left[B_{0}\right]$ is connected, we must have $B_{0} \subseteq V X$.

We infer that $X$ is the only component of $G-V D$, and that $\delta(V D)$ is therefore a bond. Since $C_{0} \in \mathcal{C}, C_{j} \in \mathcal{C}, C_{0} \cap C_{j}=\emptyset$ and $\delta(V D) \subseteq C_{0} \cup C_{j}$, the strictness of $C$ is contradicted. Hence either $C_{j}-C_{0} \subseteq\left[A_{0}, A_{0}\right]$ or $C_{j}-C_{0} \subseteq\left[B_{0}, B_{0}\right]$. 
Case II. Suppose $j \in\{1, n-1\}$. By Lemma 3, we may assume without loss of generality that $j=1$. Since $C_{0} \neq C_{1}$ (otherwise $C_{0} \cap C_{1} \cap C_{2} \neq \emptyset$ ), we may further assume without loss of generality that $C_{1} \cap\left[A_{0}, A_{0}\right] \neq \emptyset$. Then by Lemma $2,\left[A_{0}, A_{0}\right]$ contains a $C_{1}$-chord of $C_{0}$. Similarly if $C_{1} \cap\left[B_{0}, B_{0}\right] \neq \emptyset$, then $\left[B_{0}, B_{0}\right]$ contains a $C_{1}-$ chord of $C_{0}$. Since $C$ is elegant, there is only one such chord, and so we cannot have both $C_{1} \cap\left[A_{0}, A_{0}\right] \neq \emptyset$ and $C_{1} \cap\left[B_{0}, B_{0}\right] \neq \emptyset$. Thus either $C_{1}-C_{0} \subseteq\left[A_{0}, A_{0}\right]$ or $C_{1}-C_{0} \subseteq\left[B_{0}, B_{0}\right]$.

The lemma has now been proved if $k=j$; let us therefore assume as an induction hypothesis that $j<k<n$ and $\left(C_{j} \cup C_{k-1}\right)-C_{0} \subseteq\left[A_{0}, A_{0}\right]$. Since $C_{k} \cap\left(C_{k-1}-C_{0}\right) \neq \emptyset$, we have $C_{k} \cap\left[A_{0}, A_{0}\right] \neq \emptyset$, and the previous result with $j$ replaced by $k$ shows that $C_{k}-C_{0} \subseteq\left[A_{0}, A_{0}\right]$. Thus $\left(C_{j} \cup C_{k}\right)-C_{0} \subseteq\left[A_{0}, A_{0}\right]$. Similarly, $\left(C_{j} \cup C_{k}\right)-C_{0} \subseteq\left[B_{0}, B_{0}\right]$ if $\left(C_{j} \cup C_{k-1}\right)-C_{0} \subseteq\left[B_{0}, B_{0}\right]$.

\section{Proof of the theorem}

Let the edges of $G$ be oriented so that every bond in $C$ is directed. Choose any $C_{i} \in C$. By Lemma 4, we may assume without loss of generality that $C_{i+1}-C_{i} \subseteq\left[A_{i}, A_{i}\right]$. We shall define $C_{i}$ to be positive if its edges are directed toward $A_{i}$ and negative otherwise. Let us assume without loss of generality that $C_{i}$ is positive. It now suffices to show that $C_{i+1}$ is negative, for then the bonds in $\mathcal{C}$ must alternate in sign, so that $C$ must be even. Without loss of generality, let $C_{i}-C_{i+1} \subseteq\left[A_{i+1}, A_{i+1}\right]$. Then $B_{i} \subseteq A_{i+1}$ since $C_{i+1} \cap\left[B_{i}, B_{i}\right]=\emptyset$. Since $C_{i} \cap C_{i+1} \neq \emptyset$ and $C_{i}$ is positive, the edges of $C_{i+1}$ must be directed toward $B_{i+1}$. Since $c_{i}-C_{i+1} \subseteq\left[A_{i+1}, A_{i+1}\right]$, Lemma 4 shows that $C_{i+2}-C_{i+1} \subseteq\left[A_{i+1}, A_{i+1}\right]$. It follows that $c_{i+1}$ is negative and the theorem is proved. 


\section{References}

[1] C.C. Chen, "On a characterization of planar graphs", Bull. Austral. Math. Soc. 24 (1981), 289-294.

[2] D.A. Holton and C.H.C. Little, "Elegant odd rings and non-planar graphs", Combinatorial Mathematics VIII, 234-268 (Proc. Eighth Australian Conf. Combinatorial Mathematics, 1980. Lecture Notes in Mathematics, 884. Springer-Verlag, Berlin, Heidelberg, New York, 1981).

Department of Mathematics and Statistics, Massey University,

Palmerston North,

New Zeal and. 